\title{
Julien Ries (éd.), Crises, ruptures, mutations dans les
} traditions religieuses

Turnhout, Brepols, 2005, 328 p.

\section{Daniel Vidal}

\section{OpenEdition}

\section{Journals}

Édition électronique

URL : http://journals.openedition.org/assr/7022

DOI : $10.4000 /$ assr.7022

ISSN : 1777-5825

\section{Éditeur}

Éditions de l'EHESS

Édition imprimée

Date de publication : 1 juin 2007

Pagination : $97-251$

ISBN : 978-2-7132-2143-9

ISSN : 0335-5985

\section{Référence électronique}

Daniel Vidal, « Julien Ries (éd.), Crises, ruptures, mutations dans les traditions religieuses », Archives de sciences sociales des religions [En ligne], 138 | avril - juin 2007, document 138-76, mis en ligne le 17 septembre 2007, consulté le 21 septembre 2020. URL : http://journals.openedition.org/assr/7022 ; DOI : https://doi.org/10.4000/assr.7022

Ce document a été généré automatiquement le 21 septembre 2020

(C) Archives de sciences sociales des religions 


\title{
Julien Ries (éd.), Crises, ruptures, mutations dans les traditions religieuses
}

Turnhout, Brepols, 2005, 328 p.

\author{
Daniel Vidal
}

1 Peut-on penser une tradition religieuse se maintenant sur une très longue durée, comme un ensemble de croyances et de pratiques telles qu'en elles-mêmes se reconduisant, fut-ce au prix d'accommodements à la marge ? Les études rassemblées en cet ouvrage (Édition originale, Crisi, Rotture e Cambiamenti, Milan, Jaca Book, 1995 ; traduction espagnole, Crisis, Rupturas y Cambios, Madrid, Trotta, 2001. Les contributions à l'édition française ont été « actualisées »), récusent d'emblée une telle interprétation, qui inscrirait les religions en un espace à l'abri des tensions et conflits culturels et politiques traversant, et travaillant, le monde autour d'elles. Sans doute, à utiliser sans précaution ni autre forme de procès le terme même de tradition, pourrait-on faire contresens, si l'on n'y distinguait ce qu'il exprime de transition et de traversée d'épreuves et de bouleversements, dont la connaissance est d'autant plus précieuse qu'elle formule à nouveaux frais la raison au fondement de ce que les auteurs nomment l'homo religiosus. L'ouvrage ne prend en compte que les religions monothéistes - d'Asie, d'Orient proche, et d'Europe. Ce n'est pas choix dicté par la facilité, au contraire : les polythéismes auraient sans doute présenté des lignes de fractures et des impératifs de réorganisation interne plus aisément repérables. Mais il convenait, selon l'esprit général qui gouverne ces contributions, de définir, au cœur même de ce qui paraît le plus pérenne, les multiples facteurs de dérives et de dissensions, de crises et de mutations.

2 En amont des systèmes religieux analysés, toujours une rupture, que rappellent les auteurs. Religion historique, le bouddhisme se situe « dans la continuité des traditions védiques et brahmaniques ", mais, en rejetant, par exemple, «l'affirmation d'un "sujet" transcendant l'ordre phénoménal ", en relativisant la notion de divin, en contestant la valeur du sacrifice, en s'élevant contre le système des castes, il subvertit et révolutionne les Upanishad, sans pour autant stabiliser son propre ensemble doctrinal. Dom Pierre Massein, qui livre sur ce sujet une réflexion passionnante, peut alors faire 
valoir les différentes étapes, souvent considérées comme autant de ruptures, que le bouddhisme va franchir, et les différents modes d'inculturation qui en diversifient en profondeur le régime de sens, et les conduites requises de s'y conformer. L'introduction du bouddhisme en Chine est l'exemple le plus achevé de ces transformations internes, qui, sans aller jusqu'à des mutations radicales, ouvrent cette "tradition" à une historicité dé-concertante. Julien Ries souligne le rôle des « fondateurs religieux » dans la genèse et le déploiement des religions. Bouddha, à l'évidence. Et Zarathustra, opérant "une profonde modification du sacré de l'Iran archaïque", prohibant tout sacrifice sanglant, introduisant la foi « en un Dieu transcendant », substituant le culte du feu - métaphore de la lumière - à l'ancien ritualisme indo-européen, et la conception d'un temps «linéaire " à la convocation d'un temps «cyclique». Et Confucius: Anne Cheng («L'homme et la société selon l'expérience confucéenne ») rappelle l'importance du culte des ancêtres dans la civilisation de la Chine antique, et la continuité qui s'établit entre le monde « céleste » et le monde proprement « humain ». La "divinité suprême " n'est pas conçue comme transcendance créatrice à laquelle l'homme se relie par un acte de croyance, mais comme instance d'ordre. C'est précisément lorsque l'ordre social progressivement se délite, à partir du viII siècle av. J.-C., que Confucius, deux siècles plus tard, prône le retour à la Voie (Dao), mais détachée de l'antique sens sacrificiel, pour être appropriée par chacun comme " attitude intériorisée ", gouvernant le rapport à soi-même et à autrui, la conscience de soi et le respect de tout autre. L'être humain est impensable s'il n'est aussitôt rapport inter-individuel, centre et raison de cercles de plus en plus larges de "sociabilité", jusqu'à conjoindre Ciel et Homme. Si « être religieux est être profondément humain ", alors se produit une mutation essentielle: de la sphère "religieuse ", le sacré est transféré à la sphère de l'homme. La spiritualité devient «l'expérience personnelle intense du sacré», le "Ciel» nommant «la possibilité infinie pour l'homme d'être toujours plus humain».

Qu'en est-il des mutations et crises dans les "religions dualistes »? Soit, au seuil du christianisme, l'orphisme, qu'analyse Dario M. Cosi en termes d'« effervescence mystique (...) sur les plans divin et humain », en rupture avec « la stabilité immuable du panorama religieux olympien traditionnel». Contre cette tradition, le "personnage d'Orphée" se construit comme renversement des valeurs codifiées, sans que soit cependant condamnée toute pratique rituelle. L'anthropogonie orphique institue une humanité "démocratique", enracinée dans le "divin", et organisée selon un axe vertical reliant l'Un (divin) et la multiple (l'homme quelconque). En ce «multiple » se manifeste la présence « temporaire mais tempétueuse (...) de cet élément divin déchu ». La raison de cette "chute»: non pas le "péché originel», mais une "faute antécédente » - une faille, le bris de l'unité primordiale, ce que D. Cosi déchiffre comme " démembrement de Dionysos par les Titans ». Au principe « unitaire » du bien, répond alors le principe du mal comme multiple, comme nombre, et ce mal « est au cœur de la société civile, et enseveli au cœur de tout homme». Là prend forme impérative l'interdit orphique de la sexualité et de l'alimentation charnelle : multiplier ne s'entend que du «mal». L'encratisme, fondé sur l'abstinence, continuera cette tradition, en la radicalisant. Et cette question lancinante du mal se situe au centre de toute religion dualiste, et en la parole de tout "prophète ». Aux frontières du mazdéisme et du christianisme, Mani (216-277) : «Je suis venu du pays de Babylone pour faire résonner un cri par le monde entier ». Julien Ries cite cette clameur ("La gnose de Mani»), et ce cri est cri de séparation des deux royaumes - Lumière/Ténèbre. Naissance, ici, du 
manichéisme, défi lancé aux Églises se constituant au nom de Jésus. Mais Mani se dit « restaurateur de l'Église de Jésus » et que par lui le Paraclet se révèle - et qu'il est, pour finir, le Paraclet. Mise en crise du corps de doctrine lentement élaboré par le christianisme : en rupture avec l'interprétation de la Genèse, «l'Homme primordial » est un précipité d'« étincelles de lumière ». En Adam, qui « vient » après, lumière aussi abonde, mais le premier homme est aveuglé par la Matière, «inconscient et égaré » jusqu'à « oublier son origine divine ». Le « monde » ainsi est irrémédiablement scindé : un principe de pure lumière / un monde où le mal se consomme. L'Église manichéenne qui s'instaure sur ces bases se fondera sur trois interdits - les trois sceaux : de la bouche (interdit du mensonge); des mains (prohibition du meurtre); du sein (interdit de reproduction). Saint Augustin fut de cette église, avant d'en être son plus farouche contempteur. Comment accepter la conception du monde comme « prison dans laquelle les démons enchaînent la Lumière »; l'inutilité de la croyance en la crucifixion, Jésus étant «crucifié dans la matière "; la prétention de Mani d'être "l'image visible en ce monde du Paraclet céleste invisible ", etc. ? Deux traditions religieuses s'affrontent autour d'un même enjeu. Cet enjeu : le mal et sa connaissance. Mani est exécuté en 277, et, en 302 viendra l'ordre de détruire les manichéens, et d'« arracher jusque dans ses racines ce fléau de perversion ». Mutation radicale !

Religions antiques, traditions débutantes: crises et ruptures en amont, crises et bouleversements en leur parcours. Le christianisme naît et se développe selon d'identiques tourmentes. Si l'examen des crises traversées par la "tradition" chrétienne ne prend pas en compte, sauf allusivement, l'Église "primitive ", non plus que l'époque médiévale, la Renaissance et ses défis, les Lumières et leurs leçons font l'objet d'études approfondies. Jean-Claude Margolin, analysant la situation de "l'homme dans le miroir de l'humanisme au temps de la Renaissance ", note l'essor des savoirs et de la "sagesse ", l'amour des livres et des trésors de connaissances qui y sont déposés. Le $\mathrm{xVI}^{\mathrm{e}}$ siècle est bien le siècle de l'« humanisme militant »: où l'homme, au centre du savoir, doit, selon Charles de Bovelles, «demeurer ferme en son humanité », qui l'institue miroir du monde et miroir de Dieu. Un anthropocentrisme se noue à un théocentrisme, le monde profane au monde "éthico-religieux ", sans que l'on puisse décider d'une solution de continuité. Tout est " passage, changement, transformation ", et, avec Juan Luis Vivès, la « sagesse » ressort de l'ordre du sacré, quand les «lettres profanes » se christianisent. Retour en force du sacré ? L'affaire est plus complexe : en l'œuvre de Cardan, philosophe et médecin italien, Henri Busson identifie un condensé de la sagesse païenne, quand J.-Cl. Margolin y décèle une sagesse civile, au croisement d'une science politique et d'une éthique où quelque sentiment de sacré semble pouvoir se dire. Montaigne pose la pensée de la mort au principe même de la sagesse, et fonde sa conduite sur une " foi rationnelle ». Érasme est sans conteste la figure emblématique de l'humaniste chrétien: "les hommes ne naissent pas hommes, ils le deviennent». C'est dire aussi bien que l'homme tient sa grandeur et sa dignité de sa liberté. De sa responsabilité : Pic de la Mirandole en son adresse à Adam : «Je t'ai installé au milieu du monde afin que de là tu examines plus commodément tout ce qui existe dans le monde. Nous ne t'avons fait ni céleste ni terrestre, ni mortel ni immortel, afin que, maître de toi-même (...) tu te composes la forme que tu aurais préférée ».

5 Sagesse, savoir, liberté: les traditions chrétiennes rencontrent là d'extrêmes objections, en partie nées en leur sein, mais très vite pouvant désorganiser les ensembles doctrinaux les plus fermes. Luther rompt avec Rome, et une neuve liberté peut se lever : libre examen des Écritures, la foi seule comme attestation de Dieu, - mais 
aussi bien une conception de l'homme comme "créature assujettie au Diable ", que seule peut sauver la grâce de Dieu. Une véritable " anthropologie de l'homme déchu » s'énonce, qui n'est pas sans rappeler les anciennes conceptions fondées sur la raison du mal originel. Rupture avec l'humanisme érasmien. Calvin assume cette rupture, mais en formule différemment les conséquences. La misère intrinsèque de l'homme s'oppose à la grandeur de l'homme habité du divin : il est « en l'esprit humain, une inclination naturelle, quelque sentiment de divinité ». Ainsi, les jeux ne sont pas faits ; mais nul ne peut «atteindre» Dieu s'il n'est porté à désespérance. J.-Cl. Margolin propose une réflexion, qui peut n'être pas immédiatement recevable, sur les modalités mises en œuvre par Rome pour une réappropriation/détournement des schèmes Renaissants. La perspective ordonne sans doute l'espace à partir du regard de l'homme, mais l'art a moins pour fonction "de diviniser l'homme que d'humaniser le divin ». L'étude "d'apparence purement mathématique » du corps humain (l'« homme de Vitruve », ou l'« homme de Vinci ») constituerait, «si je puis dire, écrit Margolin, une sacralisation rationnelle de l'homme», exprimant "l'harmonie divine du monde». Les portraits? Érasme par Quentin Metsys, Dürer par lui-même? : une "sacralisation du profane ou une christianisation du paganisme " - ainsi qu'il en va pour la lecture "sublimée » d'Ovide. Mais c'est à juste titre que l'historien, spécialiste éminent d'Érasme et des études sur la Renaissance, souligne l'intériorisation de l'éloquence, qui ouvre la voie à la méditation intérieure, que reprennent à leur compte mystiques et « saints » tout au long du XVII ${ }^{\mathrm{e}}$ siècle. Certes, la Renaissance posa, à l'Église de Rome, de redoutables défis, dont le moindre ne fut pas son "optimisme » fondé sur un argumentaire de liberté et de responsabilité de l'homme. Si la Réforme développa une conception radicalement différente, en un "pessimisme " sans autre recours que la grâce, tous les humanistes convinrent que le tableau comportait plus d'une «plage d'ombre ». Starobinski disait la Renaissance "l'âge d'or de la mélancolie». Guillaume Budé, que J.-Cl. Margolin présente comme «le symbole même du renouveau et de l'enthousiasme intellectuel et spirituel » n'écrivait-il pas : «Quant à moi, je suis plutôt enclin à penser que le dernier jour a commencé à tomber, et que le monde est déjà au déclin ; qu'il est vraiment vieux et privé de sens, qu'il indique, présage et annonce, sa fin prochaine et sa chute»? L'extrême actualité du propos pourrait-elle nous échapper?

6 Autres temps, autres mises en crise des «traditions religieuses". Le XVIII siècle et ses Lumières, qu'analyse Jean-Pierre Sironneau ( «La crise religieuse des Lumières et la sécularisation») confirment, ou aggravent, l'effondrement de ce que Peter Berger nommait les "structures de crédibilité », ce qui conduisait Georges Gusdorf à dire que ce siècle était celui de la "laïcisation ». Les nouvelle sources de certitudes - séculières mais aussi bien religieuses - ne sont plus à chercher en un rapport de croyance à une quelconque "transcendance", mais dans la conscience individuelle. La sécularisation marque en effet une rupture, majeure, avec la tradition. Pratiques religieuses, valeurs normatives, entrent en "récession», selon l'expression de J.-P. Sironneau. Le déploiement $\mathrm{du}$ rationalisme en toutes sphères de l'activité humaine, porte contestation au cœur même de la foi, au bénéfice d'un libre examen qui ne laisse plus de place aux représentations illusoires. Kant: "Aie le courage de ton propre entendement", car il relève de la raison. Face à celle-ci, le christianisme peut en appeler à Pascal, qui distingue l'autonomie totale du discours scientifique, de l'autorité du droit et de la théologie. Mais dès lors que s'effectue la critique rationnelle de la Bible, et que s'opère l'analyse des critères de la "vérité » religieuse, ce ne sont pas seulement des « régimes » entiers de religion qui, à l'exemple des Quakers, entrent en 
dissidence. Mais des mutations plus importantes se produisent, accompagnant, dans le sillage de Spinoza et de Bayle, le développement de la méthode historico-critique : un "christianisme éclairé» se propose, et piétisme, déisme et théisme se portent progressivement aux frontières, ou en exil, de l'espace chrétien. La question de l'historicité des récits bibliques (Lessing, Herder) s'oppose à la conception chrétienne de l'intemporalité du Texte. Le problème du mal fait à nouveau surface, qui avait constitué, on l'a vu, l'un des thèmes les plus conflictuels au sein des religions antiques. Quand Rousseau indexe le mal, et la corruption, à la « société ", Kant le luthérien lie le mal à la liberté de l'homme, et en fonde en « raison » l'origine. De « Dieu » à l'homme le mal est transféré. Dès lors, non seulement un " déisme rationaliste » peut se formuler, qui agrège liberté, tolérance et assise «naturelle» de la «religion» (Locke, mais surtout Toland, trop méconnu); mais une philosophie de l'histoire s'énonce, désacralisant le temps de la théologie chrétienne. Car le temps historique est l'affaire de l'homme. Kant définit l'histoire, rappelle J.-P. Sironneau, comme « auto-avènement du genre humain vers plus de moralité et de raison », quand Herder concilie, en une configuration, promise, pour le meilleur ou pour le pire, à un bel avenir, « progressisme des Lumières " et "singularité de chaque peuple ", et que Hegel pense l'histoire comme " calvaire de l'absolu », Dieu ne se manifestant «vraiment qu'en sa fin ». La "raison » des Lumières est ainsi, paradoxalement, grosse d'une eschatologie... De là, en partie, ses limites. Le piétisme en vient, qui se conjugue en effet au rationalisme dans la défense de la conscience individuelle, mais s'en sépare sur la question de la foi, vécu émotionnel et subjectif. À l'évidence, l'homo ideologicus se substitue à l'homo religiosus, le citoyen au sujet croyant. Mais on sait les échecs de toute tentative d'instaurer une "religion civile ». Si «transfert de sacralité » il y eut, c'est moins en cette tentative, qu'en la fondation de ce que Raymond Aron appelait une « religion politique », où l'emprise du sacré est reversée au compte du séculier.

7 Mais doit-on pour autant, avec J.-P. Sironneau, créditer la « raison » de tous les péchés «totalitaires» du monde? «La » raison n'est-elle pas un signifiant assez flottant, et pluriel, pour qu'elle soit l'« objet » d'un examen plus attentif et diversifié ? Tous les totalitarismes adviennent-ils sur fond d'une " raison » au sens partiel retenu en cette analyse? De ce sens nécessairement contraint, retenons cependant ici ce qui apparaît en effet comme la plus forte injonction de mutation que puisse rencontrer, aujourd'hui, le christianisme comme tradition. D’emblée, le défi du «désenchantement», que Marcel Gauchet définit comme "l'avenir logique de la religion", et que porte la religion comme en elle-même son instance tragique, et irréversible, de déconstruction. Et le refus de toute "resacralisation", argumenté par Paul Ladrière à l'encontre de toute idéologie du "retour du religieux» ou de toute survenue de "nouveaux mouvements religieux émotionnels » conçus comme révélateurs de «la faillite de la raison moderne (..), décompresseurs providentiels d'une rationalisation excessive de la société ». Afin, poursuit P. Ladrière, "que soit définitivement assuré l'espace de l'autonomie humaine", et que soit "achevée la tâche rationalisatrice d'un christianisme désacralisant ", il faut - ultime défi objecté, assigné, peut-être? aux traditions religieuses -, que la «transcendance divine » soit résolument "arrachée à l'immanence du sacré ». Un immense champ de ruptures et de crises, de ruines aussi, s'ouvre ainsi, au vif de l'histoire présente. 